\title{
UNIVERSALITY TYPE LIMITS FOR BERGMAN ORTHOGONAL POLYNOMIALS
}

\author{
DORON S. LUBINSKY
}

\begin{abstract}
We establish universality type limits for Bergman orthogonal polynomials on simply connected regions in the complex plane with smooth boundary.
\end{abstract}

Keywords: Universality Limits, Bergman Polynomials

AMS Classification: 41A55, 65D30, 65B99, 42C99

Research supported by NSF grant DMS0700427 and US-Israel BSF grant 2004353

\section{Introduction and Results}

Let $G$ be a bounded simply connected domain in the complex plane, bounded by a Jordan curve $\Gamma$. Let $\mu$ be a finite positive Borel measure on $G$. We may define, for $n \geq 0$, orthonormal polynomials

$$
p_{n}(z)=\kappa_{n} z^{n}+\ldots, \kappa_{n}>0
$$

satisfying

$$
\int_{G} p_{n} \overline{p_{m}} d \mu=\delta_{m n}
$$

We shall usually assume that $\mu$ is regular in the sense of Stahl and Totik $[27$, p. 60$]$, so that

$$
\lim _{n \rightarrow \infty} \kappa_{n}^{1 / n}=\frac{1}{\operatorname{cap}(G)} .
$$

Here $\operatorname{cap}(G)$ denotes the logarithmic capacity of $G$. Moreover, we shall also assume that $\mu$ is absolutely continuous with respect to planar Lebesgue measure $d A$ near given points on $\partial G$. In this sense, the polynomials $\left\{p_{n}\right\}$ fall within the framework of Bergman polynomials. Throughout, we let

$$
w(z)=\frac{d \mu}{d A}(z), \text { a.e. } z \in G .
$$

Moreover, for $u \in \partial G$, we define

$$
w(u)=\lim _{z \rightarrow u, z \in G} w(z),
$$

whenever the limit is defined. We note that when $w>0$ a.e. on $G$, then it follows from Widom's criterion for regularity, that $\mu$ is regular in the sense of Stahl and Totik [27, pp. 106-107].

Date: November 3, 2009. 
There is a well developed theory of Bergman polynomials - their basic properties, and the asymptotic behavior, including that of their zeros [6], [7], [8], [12], [18], [19], [23]. In describing these, the conformal map $\phi$ of the exterior of $\Gamma$, namely

$$
D=\mathbb{C} \backslash \bar{G}
$$

onto the exterior of the unit ball plays a key role. We say that the curve $\Gamma$ is of class $C(p, \alpha)$ if the parametrization of the curve is $p$ times continuously differentiable, with $p$-th derivative satisfying a Lipschitz condition of order $\alpha \in(0,1)$.

Classical results of Suetin give asymptotics for $p_{n}$ when $\mu$ is absolutely continuous on $G$, with $w=1$ there. We shall denote the corresponding polynomials by $\left\{p_{n}^{C}\right\}$, where the $C$ stands for Carleman. It is known [28, Theorem 1.3, p. 21] that if $\Gamma \in C(1, \alpha)$, where $\alpha>\frac{1}{2}$, then uniformly for $z \in \partial G$, and even locally uniformly in $\bar{D}$,

$$
p_{n}^{C}(z)=\sqrt{\frac{n+1}{\pi}} \phi^{\prime}(z) \phi(z)^{n}\left\{1+O\left(\frac{1}{n^{2 \alpha-1}}\right)\right\} .
$$

The $n$-th reproducing kernel for $\mu$ is

$$
K_{n}(z, u)=\sum_{j=0}^{n-1} p_{j}(z) \overline{p_{j}(u)}
$$

and its normalized cousin is

$$
\tilde{K}_{n}(z, u)=w(z)^{1 / 2} w(u)^{1 / 2} K_{n}(z, u) .
$$

Recall that $w=\frac{d \mu}{d A}$ or its limit on the boundary $\partial G$.

In formulating our result, we need the notion of the convex hull $C o(K)$ of a set $K$, as well as its boundary $\partial C o(K)$. If $J \subset \partial G$, a $\partial G$ neighborhood of $J$ means a relatively open subset $J_{1}$ of $\partial G$ containing $J$. That is, $J_{1} \supset J$ and $J_{1}=U \cap \partial G$ for some open subset $U$ of the plane. Our main result is:

\section{Theorem 1.1}

Let $G$ be a bounded simply connected set, and assume that $\Gamma=\partial G$ is of class $C(1, \alpha)$, with $\alpha \in\left(\frac{1}{2}, 1\right)$. Let $J \subset \partial G$ be compact, and let some $\partial G$ neighborhood of $J$ also lie in $\partial C o(G)$. Let $\mu$ be a finite positive Borel measure on $G$ that is regular in the sense of Stahl and Totik. Assume that $\mu$ is absolutely continuous with respect to planar Lebesgue measure in an open subset of $G$ whose boundary contains a $\partial G$ neighborhood of J. Assume moreover, that $w$ is positive and continuous at each point of $J$. Then uniformly for $z \in J$ and $a, b$ in compact subsets of the plane, we have

$$
\lim _{n \rightarrow \infty} \frac{K_{n}\left(z+\frac{a}{n}, z+\frac{b}{n}\right)}{K_{n}(z, z)}=H\left(a \phi^{\prime}(z) \overline{\phi(z)}+b \overline{\phi^{\prime}(z)} \phi(z)\right),
$$


where

$$
H(t)=\left\{\begin{aligned}
2 \frac{e^{t}(t-1)+1}{t^{2}}, & t \neq 0 \\
1, & t=0
\end{aligned}\right.
$$

\section{Remarks}

(a) Universality limits for measures with support on the real line take the form

$$
\lim _{n \rightarrow \infty} \frac{K_{n}\left(\xi+\frac{a}{\tilde{K}_{n}(\xi, \xi)}, \xi+\frac{b}{\tilde{K}_{n}(\xi, \xi)}\right)}{K_{n}(\xi, \xi)}=\frac{\sin \pi(a-b)}{\pi(a-b)} .
$$

Here $\xi$ is in the "bulk" of the support, that is, $\xi$ lies in the interior of $\operatorname{supp}[\mu]$. See [2], [3], [4], [5], [9], [11], [13], [14], [15], [17], [25], [26], [30]. For measures supported on the unit circle, the analogous formulation is [10]

$$
\lim _{n \rightarrow \infty} \frac{1}{n} \tilde{K}_{n}\left(e^{i\left(\theta+\frac{2 \pi a}{n}\right)}, e^{i\left(\theta+\frac{2 \pi b}{n}\right)}\right)=e^{i \pi(a-b)} \frac{\sin \pi(a-b)}{\pi(a-b)}
$$

or

$$
\lim _{n \rightarrow \infty} \frac{K_{n}\left(z\left(1+\frac{i 2 \pi a}{n}\right), z\left(1+\frac{i 2 \pi \bar{b}}{n}\right)\right)}{K_{n}(z, z)}=e^{i \pi(a-b)} \frac{\sin \pi(a-b)}{\pi(a-b)},
$$

uniformly for $a, b$ in compact subsets of the complex plane and $z=e^{i \theta}, \theta \in J$. (b) Note that the increment inside the reproducing kernel in (1.5) is $\frac{a}{n}$ or $\frac{b}{n}$. By contrast, under the hypotheses of Theorem 1.1, $K_{n}(z, z)$ grows like $n^{2}$, and

$$
\lim _{n \rightarrow \infty} \frac{K_{n}\left(z+\frac{a}{K_{n}(z, z)}, z+\frac{b}{K_{n}(z, z)}\right)}{K_{n}(z, z)}=1 .
$$

(c) The restriction that $J \subset \partial G \cap \partial C o(G)$ is a severe geometric restriction - basically requiring that $G$ is "locally convex" in some neighborhood of $J$. It is likely that there is some payoff between the generality of the measure $\mu$ and the geometry of the domain $G$. In particular, for Carleman polynomials, there is no need for a geometric restriction, as shown in Section 2. We use the convexity in constructing, for each $z \in J$, a polynomial $R_{z}$, such that $R_{z}(z)=1$ and $\left|R_{z}\right|<1$ in $\bar{G} \backslash\{z\}$. To allow for more general sets $J$, we need a definition:

Definition 1.2 Let $S \subset \mathbb{C}$ be bounded.

(a) We say that $a \in S$ is a peak polynomial point for $S$ if there exists a polynomial $R_{a}$ such that

$$
R_{a}(a)=1
$$

and

$$
\left|R_{a}\right|<1 \text { in } S \backslash\{a\} .
$$

(b) Let $J \subset S$. We say that $J$ is a uniform peak polynomial set for $S$ if each point $a \in J$ is a peak polynomial point for $S$, and moreover, the 
degree of $R_{a}$ is bounded above independent of $a \in J$, and in addition, for any $\delta>0$, there exists $r<1$ independent of $a$, such that

$$
\left|R_{a}(z)\right|<r \text { for } a \in J \text { and } z \in G \text { with }|z-a| \geq \delta .
$$

\section{Remark}

Peak polynomial points have been investigated (without using that name) by Nagy and Totik [20]. Uniform peak polynomial sets have been discussed by Andrievskii and Pritsker [1]. In particular, they derived a necessary and sufficient condition involving circles that lie in $\mathbb{C} \backslash G$ touching $\partial G$ at only one point. This overlaps with results of Totik [20], [31].

\section{Theorem 1.3}

Assume the hypotheses of Theorem 1.1, except the restriction that some $\partial G$ neighborhood of $J$ also lies in $\partial C o(G)$. Assume instead that some $\partial G$ neighborhood of $J$ is a uniform peak polynomial set for $\bar{G}$. Then the conclusion of Theorem 1.1 remains valid.

\section{Remark}

Totik [31] has recently established asymptotics for Christoffel functions associated with Bergman polynomials over a region that consists of finitely many domains. Totik does not require a geometric condition on the boundary, but does require a locally $C^{2}$ boundary. This should lead to a version of Theorem 1.3 without geometric conditions, but with a locally smoother boundary.

\section{Corollary 1.4}

Assume the hypotheses of Theorem 1.1 or 1.3. Let $r, s$ be non-negative integers and

$$
K_{n}^{(r, s)}(z, z)=\sum_{k=0}^{n-1} p_{k}^{(r)}(z) \overline{p_{k}^{(s)}(z)} .
$$

Then uniformly for $z \in J$,

$$
\lim _{n \rightarrow \infty} \frac{K_{n}^{(r, s)}(z, z)}{n^{r+s} K_{n}(z, z)}=\frac{2\left(\phi^{\prime}(z) \overline{\phi(z)}\right)^{r}\left(\overline{\phi^{\prime}(z)} \phi(z)\right)^{s}}{r+s+2} .
$$

In the sequel $C, C_{1}, C_{2}, \ldots$ denote constants independent of $n, z, u, v, s, t$. The same symbol does not necessarily denote the same constant in different occurences. We shall write $C=C(\alpha)$ or $C \neq C(\alpha)$ to respectively denote dependence on, or independence of, the parameter $\alpha$. Given measures $\mu^{*}$, $\mu^{\#}$, we use $K_{n}^{*}, K_{n}^{\#}$ and $p_{n}^{*}, p_{n}^{\#}$ to denote respectively their reproducing kernels and orthonormal polynomials. Similarly superscripts $*$,\# are used to distinguish other quantities associated with them. The superscript $C$ (for 
Carleman) denotes quantities associated with the Legendre weight 1 on $G$. For $z \in \mathbb{C}$ and $\delta>0$, we set

$$
B(z, \delta)=\{t:|t-z|<\delta\} .
$$

The distance from a point $z$ to a set of complex numbers $J$ is denoted $\operatorname{dist}(z, J)$. For such a set $J$, we set

$$
J(\delta)=\{z \in \mathbb{C}: \operatorname{dist}(z, J) \leq \delta\} .
$$

For $n \geq 1$ and $M>0$, we also let

$$
G_{n}(M)=\left\{z \in \bar{D}: \operatorname{dist}(z, \partial G) \leq \frac{M}{n}\right\}
$$

In particular, $G_{n}(M)$ contains $\partial G$. $[x]$ denotes the greatest integer $\leq x$. We denote the $n$th Christoffel function for the measure $\mu$ by

$$
\lambda_{n}(z)=1 / K_{n}(z, z)=\min _{\operatorname{deg}(P) \leq n-1} \int_{G}|P|^{2} d \mu /|P(z)|^{2} .
$$

As in [13], the main idea in this paper is a localization principle based on a comparison inequality (Lemma 4.1 below). Suppose that $\mu, \mu^{*}$ are measures with $\mu \leq \mu^{*}$ in $G$, and let the superscript $*$ indicate quantities associated with the measure $\mu^{*}$. Then for $z, t \in \mathbb{C}$,

$$
\begin{aligned}
& \left|K_{n}(z, t)-K_{n}^{*}(z, t)\right| / K_{n}(z, z) \\
\leq & \left(\frac{K_{n}(t, t)}{K_{n}(z, z)}\right)^{1 / 2}\left[1-\frac{K_{n}^{*}(z, z)}{K_{n}(z, z)}\right]^{1 / 2} \\
= & \left(\frac{\lambda_{n}(z)}{\lambda_{n}(t)}\right)^{1 / 2}\left[1-\frac{\lambda_{n}(z)}{\lambda_{n}^{*}(z)}\right]^{1 / 2} .
\end{aligned}
$$

The paper is organised as follows. In Section 2, we prove some of the results for the weight 1 on $G$, that is for Carleman polynomials. In Section 3 , we present some asymptotics for Christoffel functions. In Section 4, we prove our localization principle, and hence Theorems 1.1, 1.3 and Corollary 1.4 .

\section{Carleman Polynomials}

For the Legendre weight (or normalized Lebesgue measure) on $G$, recall that we use the superscript $C$ to distinguish the orthonormal polynomials and related quantities. We shall use the asymptotics (1.2):

\section{Theorem 2.1}

Let $G$ be a bounded simply connected set, and assume that $\Gamma=\partial G$ is of class $C(1, \alpha)$, with $\alpha \in\left(\frac{1}{2}, 1\right)$. Let $M>0$. 
(a) Uniformly for $z, t \in G_{n}(M)$, with $z \neq t$,

$$
K_{n}^{C}(z, t)=\frac{\phi^{\prime}(z) \overline{\phi^{\prime}(t)}}{\pi}\left\{-(n+1) \frac{[\phi(z) \overline{\phi(t)}]^{n}}{1-\phi(z) \overline{\phi(t)}}+\frac{1-[\phi(z) \overline{\phi(t)}]^{n+1}}{[1-\phi(z) \overline{\phi(t)}]^{2}}\right\}+O\left(n^{3-2 \alpha}\right) .
$$

(b) Uniformly for $z \in \partial G$,

$$
K_{n}^{C}(z, z)=\frac{\left|\phi^{\prime}(z)\right|^{2}}{\pi} \frac{n(n+1)}{2}+O\left(n^{3-2 \alpha}\right) .
$$

Moreover, uniformly for $z \in G_{n}(M)$,

$$
K_{n}^{C}(z, z)=O\left(n^{2}\right) .
$$

(c) Uniformly for $z \in \partial G$ and $a, b$ in compact subsets of the plane,

$$
\lim _{n \rightarrow \infty} \frac{K_{n}^{C}\left(z+\frac{a}{n}, z+\frac{b}{n}\right)}{K_{n}^{C}(z, z)}=H\left(a \phi^{\prime}(z) \overline{\phi(z)}+\overline{b \phi^{\prime}(z)} \phi(z)\right) .
$$

Proof

Let $v \in G_{n}(M)$. We can write $v=z+\frac{a}{n}$, where $z \in \partial G$ and $|a| \leq M$. Then

$$
\phi(v)-\phi(z)=O(|v-z|)=O\left(\frac{1}{n}\right),
$$

for $n \geq n_{0}(M)$. Here we have used the fact that $\Gamma=\partial G$ is of class $C(1, \alpha)$, while $\phi^{\prime}$ is continuous and non-zero on $\partial G$, so that every point $v$ in $G_{n}(M)$ may be joined to a point $z$ in $\partial G$, by a path of length $O(|v-z|)$. As $|\phi(z)|=1$, we obtain

$$
|\phi(v)| \leq 1+\frac{C}{n},
$$

and hence

$$
\sup _{v \in G_{n}(M)} \sup _{1 \leq k \leq n}\left|\phi^{k}(v)\right| \leq C .
$$

We also note that then if $P$ is a polynomial of degree $\leq n$, the WalshBernstein inequality, namely

$$
|P(z)| \leq|\phi(z)|^{n} \sup _{\partial G}|P|
$$

gives

$$
\sup _{G_{n}(M)}|P| \leq C \sup _{\partial G}|P| .
$$

(a) From (1.2), uniformly for $z, t \in G_{n}(M)$,

$$
\begin{aligned}
K_{n}^{C}(z, t) & =\frac{\phi^{\prime}(z) \overline{\phi^{\prime}(t)}}{\pi} \sum_{k=0}^{n-1}(k+1)[\phi(z) \overline{\phi(t)}]^{k}\left\{1+O\left(\frac{1}{k^{2 \alpha-1}}\right)\right\} \\
& =\frac{\phi^{\prime}(z) \overline{\phi^{\prime}(t)}}{\pi} \sum_{k=0}^{n-1}(k+1)[\phi(z) \overline{\phi(t)}]^{k}+O\left(\sum_{k=0}^{n-1} k^{2-2 \alpha}\right)
\end{aligned}
$$


recall (2.5). Using the identity

$$
\sum_{k=0}^{n-1}(k+1) u^{k}=-(n+1) \frac{u^{n}}{1-u}+\frac{1-u^{n+1}}{(1-u)^{2}}, u \neq 1,
$$

(this is easily obtained by differentiating the identity for a finite geometric series), and the fact that $\alpha>\frac{1}{2}$, we obtain (2.1).

(b) This is similar to (a), but easier.

(c) Suppose first that $z+\frac{a}{n}$ and $z+\frac{b}{n} \in \bar{D}$, so that $\phi\left(z+\frac{a}{n}\right)$ and $\phi\left(z+\frac{b}{n}\right)$ are well defined. We have for some path $\gamma$ in $\bar{D}$ from $z$ to $z+\frac{a}{n}$,

$$
\phi\left(z+\frac{a}{n}\right)-\phi(z)-\frac{a}{n} \phi^{\prime}(z)=\int_{\gamma}\left(\phi^{\prime}(t)-\phi^{\prime}(z)\right) d t=o\left(\frac{1}{n}\right),
$$

uniformly for $z \in \partial G$, and $a$ in compact subsets of the complex plane, because of the continuity of $\phi^{\prime}$. Let

$$
\Delta=a \phi^{\prime}(z) \overline{\phi(z)}+\overline{b \phi^{\prime}(z)} \phi(z) .
$$

Then, as $|\phi(z)|=1$,

$$
\begin{gathered}
\phi\left(z+\frac{a}{n}\right) \overline{\phi\left(z+\frac{b}{n}\right)}=1+\frac{\Delta}{n}+o\left(\frac{1}{n}\right) ; \\
{\left[\phi\left(z+\frac{a}{n}\right) \overline{\phi\left(z+\frac{b}{n}\right)}\right]^{n}=e^{\Delta+o(1)} .}
\end{gathered}
$$

Substituting into $(2.1)$ gives, as long as $\Delta \neq 0$, and $n$ is so large that $z+\frac{a}{n}, z+\frac{b}{n} \in G_{n}(M)$,

$$
\begin{aligned}
& K_{n}^{C}\left(z+\frac{a}{n}, z+\frac{b}{n}\right) \\
= & \frac{\phi^{\prime}\left(z+\frac{a}{n}\right) \overline{\phi^{\prime}\left(z+\frac{b}{n}\right)}}{\pi}\left\{\frac{(n+1) n e^{\Delta+o(1)}}{\Delta+o(1)}+\frac{n^{2}\left(1-e^{\Delta+o(1)}\right)}{\Delta^{2}+o(1)}\right\}
\end{aligned}
$$

and so using continuity of $\phi^{\prime}$, and (2.2),

$$
\begin{aligned}
\frac{K_{n}^{C}\left(z+\frac{a}{n}, z+\frac{b}{n}\right)}{K_{n}^{C}(z, z)} & =2\left\{\frac{e^{\Delta}}{\Delta}+\frac{1-e^{\Delta}}{\Delta^{2}}\right\}+o(1) \\
& =H(\Delta)+o(1) .
\end{aligned}
$$

We still have to show the convergence when $\Delta=0$, or when at least one of $z+\frac{a}{n}$ and $z+\frac{b}{n} \notin \bar{D}$, that is at least one is in $G$. For this we use convergence continuation theorems, and uniform boundedness. In view of Cauchy-Schwarz, (2.6) and (2.2), we have for all $z \in \partial G$,

$$
\sup _{u, v \in G_{n}(M)}\left|K_{n}^{C}(u, v)\right| \leq \sup _{u \in G_{n}(M)}\left|K_{n}^{C}(u, u)\right| \leq C n^{2} \leq C K_{n}^{C}(z, z) .
$$


The maximum modulus principle then gives

$$
\sup _{u, v \in G \cup G_{n}(M)}\left|K_{n}^{C}(u, v)\right| \leq C K_{n}^{C}(z, z) .
$$

Thus $\left\{\frac{K_{n}^{C}\left(z+\frac{a}{n}, z+\frac{b}{n}\right)}{K_{n}^{C}(z, z)}\right\}_{n}$ is uniformly bounded for $a, b$ in compact subsets of the plane, and for $z \in \partial G$. It is hence a normal family in the complex variables $a, b$. Inasmuch as for $\Delta \neq 0$, it has the limit $H\left(a \phi^{\prime}(z) \overline{\phi(z)}+\overline{b \phi^{\prime}(z)} \phi(z)\right)$, which is entire in $a, b$, convergence continuation theorems give the result, even when $\Delta=0$.

Recall the notation (1.9).

\section{Corollary 2.2}

Let $r, s$ be non-negative integers. Then uniformly for $z \in \partial G$,

$$
\lim _{n \rightarrow \infty} \frac{K_{n}^{C(r, s)}(z, z)}{n^{r+s} K_{n}(z, z)}=\frac{2\left(\phi^{\prime}(z) \overline{\phi(z)}\right)^{r}\left(\overline{\phi^{\prime}(z)} \phi(z)\right)^{s}}{r+s+2} .
$$

\section{Proof}

Taylor series expansion shows that

$$
\frac{K_{n}^{C}\left(z+\frac{a}{n}, z+\frac{b}{n}\right)}{K_{n}^{C}(z, z)}=\sum_{r, s=0}^{\infty} \frac{a^{r} \bar{b}^{s}}{r ! s !} \frac{K_{n}^{C(r, s)}(z, z)}{n^{r+s} K_{n}^{C}(z, z)} .
$$

Next, the Maclaurin series for $H$ is

$$
H(t)=\sum_{k=0}^{\infty} \frac{2 t^{k}}{(k+2) k !}
$$

so if $w=\phi^{\prime}(z) \overline{\phi(z)}$,

$$
\begin{aligned}
& H\left(a \phi^{\prime}(z) \overline{\phi(z)}+\overline{b \phi^{\prime}(z)} \phi(z)\right) \\
= & \sum_{k=0}^{\infty} \frac{2}{(k+2) k !} \sum_{r=0}^{k}\left(\begin{array}{l}
k \\
r
\end{array}\right)(a w)^{r}(\overline{b w})^{k-r} \\
= & 2 \sum_{r=0}^{\infty} \frac{(a w)^{r}}{r !} \sum_{k=r}^{\infty} \frac{(\overline{b w})^{k-r}}{(k+2)(k-r) !} \\
= & 2 \sum_{r, s=0}^{\infty} \frac{(a w)^{r}}{r !} \frac{(\overline{b w})^{s}}{s !(r+s+2)} .
\end{aligned}
$$

The uniform convergence in $a, b$ in (2.4) as $n \rightarrow \infty$, implies that corresponding Taylor series coefficients also converge. Then (2.8) and (2.9) give (2.7). The uniformity in $z$ follows from Cauchy's estimates and the uniform convergence in $z \in \partial G$. 


\section{Christoffel functions}

The methods used to prove the following result for Christoffel functions are well known for orthogonal polyonomials over intervals and curves [16], [21], [22], [29]. For Bergman polynomials, there are far fewer results [7], [8]. One difficulty in its formulation is that if $z \in \partial G$, and $a \in \mathbb{C}$, we cannot be sure that $z+\frac{a}{n} \in \partial G$ for large enough $n$. So instead we consider sequences $\left\{z_{n}\right\} \subset \partial G$. Recall that $\lambda_{n}$ and $\lambda_{n}^{C}$ denote respectively the Christoffel functions for the measure $\mu$ and for the measure with derivative 1 on $G$ (the Carleman case).

\section{Theorem 3.1}

Let $G, J$ and $\mu$ be as in Theorem 1.1 or 1.3. Let $M>0$.

(a) Uniformly for $z_{0} \in J$ and sequences $\left\{z_{n}\right\} \subset \partial G$ with $\left|z_{n}-z_{0}\right|<\frac{M}{n}, n \geq$ 1 ,

$$
\lim _{n \rightarrow \infty} n^{2} \lambda_{n}\left(z_{n}\right)=\frac{\left|\phi^{\prime}\left(z_{0}\right)\right|^{2}}{\pi} w\left(z_{0}\right) .
$$

(b) There exists $\eta>0$ and $n_{0}$ such that uniformly for $n \geq n_{0}$ and $z \in$ $J(\eta) \cap \partial G$,

$$
\lambda_{n}(z) \sim \frac{1}{n^{2}}
$$

\section{Remarks}

(a) The notation $\sim$ means that the ratio of the two quantities is bounded above and below by positive constants independent of $n, z$ and $a$.

(b) We emphasize that we are assuming that $w$ is continuous in $J$ when regarded as a function defined on $G$.

(c) Totik [31, Theorem 1.4] has recently proved a generalization of Theorem 3.1, where the region $G$ may consist of finitely many components, and moreover, there is no geometric condition on the boundary. The decription of the limit involves a Green's function rather than a conformal map. However, instead it is assumed that $J$ is contained in a $C^{2}$ arc, so the curve is assumed to be locally smoother than in our case.

We shall assume the hypotheses of Theorem 1.1, and at the end, indicate the simple changes required for the hypotheses of Theorem 1.3. We first need:

\section{Lemma 3.2}

Assume the hypotheses of Theorem 1.1 and that $J_{1}$ is a $\partial G$ neighborhood of $J$ such that $J_{1} \subset \partial G \cap \partial C o(G)$. There exists for $z \in J_{1}$, a quadratic polynomial $R_{z}$ with the following properties:

(i) $R_{z}(z)=1$;

(ii) $\left|R_{z}\right| \leq 1$ in $G$; 
(iii) Given $\delta>0$, there exists $\eta \in(0,1)$ such that $\left|R_{z}\right| \leq \eta$ in $G \backslash B\left(z, \frac{\delta}{2}\right)$. Here $\eta$ is independent of $z \in J_{1}$.

\section{Proof}

By a translation and rotation of $G$, we may assume that $\bar{G}$ lies in the closed left-half plane, and that the $z$ for which $R_{z}$ must be constructed is $z=0$. Thus the imaginary axis is a supporting line for $C o(\bar{G})$. By a dilation, we may also assume that

$$
\bar{G} \subset\left\{z:|z| \leq \frac{1}{13} \sin \frac{\pi}{8}\right\}
$$

We shall see that

$$
R_{0}(t)=1+t+2 t^{2}
$$

does the job. Let $t=r e^{i \theta}$, where $\theta \in\left[\frac{\pi}{2}, \pi\right]$ and $0 \leq r \leq \frac{1}{13} \sin \frac{\pi}{8}$. It suffices to consider such $t$, since $|R(\bar{t})|=|R(t)|$. A simple calculation shows that

$$
\begin{aligned}
& \left|R_{0}(t)\right|^{2} \\
= & 1+r\left\{2 \cos \theta+r[1+4 \cos 2 \theta]+4 r^{2} \cos \theta+4 r^{3}\right\} .
\end{aligned}
$$

If first, $\theta \in\left[\frac{\pi}{2}, \frac{5 \pi}{8}\right]$, then as $\cos \theta \leq 0$,

$$
\begin{aligned}
& \left|R_{0}(t)\right|^{2} \\
\leq & 1+r^{2}\left\{1+4 \cos 2 \theta+4 r^{2}\right\} \\
\leq & 1+r^{2}\left\{1-2 \sqrt{2}+\frac{4}{169}\right\} \\
\leq & 1-r^{2}\{\sqrt{2}-1\} .
\end{aligned}
$$

If instead, $\theta \in\left(\frac{5 \pi}{8}, \pi\right]$, then

$$
\begin{aligned}
& \left|R_{0}(t)\right|^{2} \\
\leq & 1+r\left\{-2 \sin \frac{\pi}{8}+5 r+4 r^{2}+4 r^{3}\right\} \\
\leq & 1-r \sin \frac{\pi}{8},
\end{aligned}
$$

as $5 r+4 r^{2}+4 r^{3}<13 r \leq \sin \frac{\pi}{8}$. Thus for all $t \in \bar{G}$,

$$
\left|R_{0}(t)\right|^{2} \leq \max \left\{1-r^{2}\{\sqrt{2}-1\}, 1-r \sin \frac{\pi}{8}\right\} .
$$

In particular, if $t \in G \backslash B\left(0, \frac{\delta}{2}\right)$, so that $|t| \geq \frac{\delta}{2}$, we have

$$
\left|R_{0}(t)\right|^{2} \leq \max \left\{1-\frac{\delta^{2}}{4}\{\sqrt{2}-1\}, 1-\frac{\delta}{2} \sin \frac{\pi}{8}\right\} .
$$

This latter bound can be taken as our $\eta^{2}$, since it will clearly work uniformly for $z \in J_{1}$.

\section{Remark}

Uniform peak polynomial sets were discussed in [1] and [31], though with 
different terminology. It is easy to see that some geometric restriction is needed. Indeed, let

$$
G=\{z:|z| \leq 1 \text { with } \arg (z) \in[\beta \pi, \pi] \cup[-\pi,-\beta \pi]\},
$$

where $0<\beta<\frac{\pi}{2}$. Thus $G$ is the ball center 0 , radius 1 , with a sector of width $2 \beta \pi<\pi$ removed about the positive real axis. Then there does not exist a polynomial $R_{0}$ with the properties listed above. Indeed, if

$$
R_{0}(z)=1+\gamma z+O\left(z^{2}\right),
$$

then in order that $\left|R_{0}\right|<1$ in that part of $G$ in the left-half plane close to 0 , we need $\gamma>0$. But then for $z$ in $G$ close to 0 in the right-half plane, we obtain $\left|R_{z}\right|>1$.

\section{Proof of Theorem 3.1 under the hypotheses of Theorem 1.1}

(a) Let $\varepsilon>0$ and choose $\delta>0$ such that $\mu$ is absolutely continuous with respect to planar measure in $J(\delta)$, and such that

$$
(1+\varepsilon)^{-1} \leq \frac{w(z)}{w(u)} \leq 1+\varepsilon, z, u \in J(\delta) \cap \bar{G} \text { with }|z-u| \leq \delta .
$$

(This is possible because of compactness of $J$ and continuity and positivity of $w$ at every point of $J)$. We may assume that $\delta$ is so small that $J(\delta) \cap \partial G$ is a uniform peak polynomial set for $\bar{G}$. Let us fix $z_{0} \in J, z \in B\left(z_{0}, \delta / 2\right) \cap \partial G$ and let $R_{z}$ be the quadratic polynomial of Lemma 3.2. Thus $R_{z}(z)=1$ and there exists $r<1$ such that

$$
\left|R_{z}\right| \leq r \text { in } G \backslash B\left(z, \frac{\delta}{2}\right) .
$$

Here $r$ is independent of $z$ and $z_{0}$. Let $\eta \in\left(0, \frac{1}{2}\right), n>2[\eta n]$, and consider the polynomial

$$
P(t)=\frac{K_{n-2[n \eta]}^{C}(t, z)}{K_{n-2[n \eta]}^{C}(z, z)} R_{z}(t)^{[n \eta]} .
$$

We see that $P(z)=1$, and $P$ has degree $\leq n-1$, so, using the properties of $R_{z}$, and (3.3), and recalling $\left|z-z_{0}\right|<\delta / 2$,

$$
\begin{aligned}
& \lambda_{n}(z) \\
\leq & \int_{G}|P|^{2} d \mu \\
\leq & \frac{w\left(z_{0}\right)(1+\varepsilon)}{K_{n-2[n \eta]}^{C}(z, z)^{2}} \int_{G \cap B\left(z, \frac{\delta}{2}\right)}\left|K_{n-2[n \eta]}^{C}(t, z)\right|^{2} d A(t) \\
& +\frac{\left\|K_{n-2[n \eta]}^{C}(\cdot, z)\right\|_{L_{\infty}\left(G \backslash B\left(z, \frac{\delta}{2}\right)\right)}^{2}}{K_{n-2[n \eta]}^{C}(z, z)^{2}} r_{G \backslash B\left(z, \frac{\delta}{2}\right)} d \mu .
\end{aligned}
$$


Here

$$
\begin{aligned}
& \int_{G \cap B\left(z, \frac{\delta}{2}\right)}\left|K_{n-2[n \eta]}^{C}(t, z)\right|^{2} d A(t) \\
\leq & \int_{G}\left|K_{n-2[n \eta]}^{C}(t, z)\right|^{2} d A(t)=K_{n-2[n \eta]}^{C}(z, z) .
\end{aligned}
$$

Moreover, by Cauchy-Schwarz, the maximum-modulus principle, and Theorem 2.1(b),

$$
\left\|K_{n-2[n \eta]}^{C}(\cdot, z)\right\|_{L_{\infty}(G)} \leq C n^{2}
$$

Hence, using $\lambda_{n-2[n \eta]}^{C}(z)=1 / K_{n-2[n \eta]}^{C}(z, z)$, and Theorem 2.1(b) again, (3.4) gives

$$
\begin{aligned}
& \lambda_{n}(z) / \lambda_{n-2[\eta n]}^{C}(z) \\
\leq & w\left(z_{0}\right)(1+\varepsilon)+C n^{2} r^{2[\eta n]} .
\end{aligned}
$$

This bound holds uniformly for $z \in B\left(z_{0}, \frac{\delta}{2}\right) \cap \partial G$ and $z_{0} \in J$. Now let $M>0$ and $\left\{z_{n}\right\} \subset \partial G$ with $\left|z_{n}-z_{0}\right| \leq \frac{M}{n}, n \geq 1$. We then obtain for $n \geq \frac{2 M}{\delta}$,

$$
\begin{aligned}
& \lambda_{n}\left(z_{n}\right) / \lambda_{n-2[n \eta]}^{C}\left(z_{n}\right) \\
\leq & w\left(z_{0}\right)(1+\varepsilon)+C n^{2} r^{[\eta n]} .
\end{aligned}
$$

Using the asymptotic (2.2) in Theorem 2.1(b), we deduce that

$$
\limsup _{n \rightarrow \infty} n^{2} \lambda_{n}\left(z_{n}\right) \leq \frac{2 \pi}{\left|\phi^{\prime}\left(z_{0}\right)\right|^{2}} w\left(z_{0}\right)(1+\varepsilon)(1-2 \eta)^{-2} .
$$

As $\varepsilon$ and $\eta>0$ are arbitrary, we obtain, uniformly for $z_{0} \in J$ and such sequences $\left\{z_{n}\right\}$,

$$
\limsup _{n \rightarrow \infty} n^{2} \lambda_{n}\left(z_{n}\right) \leq \frac{2 \pi}{\left|\phi^{\prime}\left(z_{0}\right)\right|^{2}} w\left(z_{0}\right) .
$$

The converse inequality is a little more difficult, and does use regularity of the measure $\mu$. Much as before, we let $R_{z}$ and $\eta$ be as above, and for $n>2[\eta n]$, we set

$$
P(t)=\frac{K_{n-2[n \eta]}(t, z)}{K_{n-2[n \eta]}(z, z)} R_{z}(t)^{[n \eta]}
$$


Much as before, we obtain

$$
\begin{aligned}
& \lambda_{n}^{C}(z) \\
\leq & \int_{G}|P|^{2} d A \\
\leq & \frac{w\left(z_{0}\right)^{-1}(1+\varepsilon)}{K_{n-2[n \eta]}(z, z)^{2}} \int_{G \cap B\left(z, \frac{\delta}{2}\right)}\left|K_{n-2[n \eta]}(t, z)\right|^{2} w(t) d A(t) \\
& +\frac{\left\|K_{n-2[n \eta]}(\cdot, z)\right\|_{L_{\infty}(G \backslash B(z, \delta / 2))}^{2}}{K_{n-2[n \eta]}(z, z)^{2}} r^{2[\eta n]} \int_{G \backslash B(z, \delta / 2)} d A .
\end{aligned}
$$

$$
\begin{aligned}
& \int_{G \cap B\left(z, \frac{\delta}{2}\right)}\left|K_{n-2[n \eta]}(t, z)\right|^{2} w(t) d A(t) \\
\leq & \int_{G}\left|K_{n-2[n \eta]}(t, z)\right|^{2} d \mu(t)=K_{n-2[n \eta]}(z, z) .
\end{aligned}
$$

Next, by the regularity of $\mu$, for any sequence $\left\{S_{n}\right\}$ of polynomials, with each $S_{n}$ of degree $\leq n$, we have [27, Thm. 3.2.1(iii), p. 66],

$$
\limsup _{n \rightarrow \infty}\left[\frac{\left\|S_{n}\right\|_{L_{\infty}(G)}}{\left(\int_{G}\left|S_{n}\right|^{2} d \mu\right)^{1 / 2}}\right]^{1 / n} \leq 1
$$

In particular,

$$
\begin{aligned}
& \left\|K_{n-2[n \eta]}(\cdot, z)\right\|_{L_{\infty}(G)}^{2} \\
\leq & (1+o(1))^{n} \int_{G}\left|K_{n-2[n \eta]}(t, z)\right|^{2} d \mu(z)=(1+o(1))^{n} K_{n-2[n \eta]}(z, z) .
\end{aligned}
$$

Substituting these estimates in (3.6) gives for such $z, z_{0}$, and $n$,

$$
\begin{aligned}
& \lambda_{n}^{C}(z) / \lambda_{n-2[n \eta]}(z) \\
\leq & w\left(z_{0}\right)^{-1}(1+\varepsilon)+(1+o(1))^{n} r^{2[\eta n]} \int_{G} d A .
\end{aligned}
$$

Now recall that $\left\{z_{n}\right\}$ is a sequence in $\partial G$ with $\left|z_{n}-z_{0}\right| \leq M / n$ for $n \geq 1$. Setting $z=z_{n-2[\eta n]}$ and using our asymptotic Theorem 2.1(b) yields

$$
\begin{aligned}
& \limsup _{n \rightarrow \infty} n^{-2} / \lambda_{n-2[n \eta]}\left(z_{n-2[n \eta]}\right) \\
\leq & \left(\frac{2 \pi}{\left|\phi^{\prime}\left(z_{0}\right)\right|^{2}} w\left(z_{0}\right)\right)^{-1}(1+\varepsilon) .
\end{aligned}
$$

Now for any $m$ large enough, we can write $m=n-2[n \eta]$ for some $n$ : indeed, the difference between successive elements, namely $(n+1-2[(n+1) \eta])-$ 
$(n-2[n \eta])=1+2([n \eta]-[(n+1) \eta])$ is no larger than 1 . We deduce that

$$
\limsup _{m \rightarrow \infty} m^{-2} / \lambda_{m}\left(z_{m}\right) \leq\left(\frac{2 \pi}{\left|\phi^{\prime}\left(z_{0}\right)\right|^{2}} w\left(z_{0}\right)\right)^{-1}(1+\varepsilon)(1-2 \eta)^{-2} .
$$

Here $\varepsilon$ and $\eta$ may be taken arbitrarily small. Together with (3.5), this gives the result.

(b) This was essentially proved in (a) - use the estimates established uniformly for $z \in B\left(z_{0}, \delta / 2\right)$ and $z_{0} \in J$.

\section{Remark}

Note that we did not use regularity of $\mu$ in the upper bound. Thus for any measure $\mu$ on $G$, (3.5) holds. Of course, this is a familiar result in the context of measures on the real line, and holds even when $w$ is not continuous at $z_{0}[16],[29]$.

Proof of Theorem 3.1 under the hypotheses of Theorem 1.3

The only difference is that we let

$$
P(t)=\frac{K_{n-k[n \eta]}^{C}(t, z)}{K_{n-k[n \eta]}^{C}(z, z)} R_{z}(t)^{[n \eta]}
$$

for the asymptotic upper bound, and

$$
P(t)=\frac{K_{n-k[n \eta]}(t, z)}{K_{n-k[n \eta]}(z, z)} R_{z}(t)^{[n \eta]}
$$

for the asymptotic lower bound, where $R_{z}$ is the polynomial of degree, say, $\leq k$ of Definition 1.2. Here $k$ is by hypothesis, independent of $z$. Moreover, $\eta \in\left(0, \frac{1}{k}\right)$. The rest of the details are as before.

\section{Proof of Theorems $1.1,1.3$ and Corollary 1.2}

We begin with an inequality:

\section{Lemma 4.1}

Suppose that $\mu, \mu^{*}$ are measures on $G$ with $\mu \leq \mu^{*}$ in $G$. Then for $z, t \in \mathbb{C}$,

$$
\begin{aligned}
& \left|K_{n}(z, t)-K_{n}^{*}(z, t)\right| / K_{n}(z, z) \\
\leq & \left(\frac{K_{n}(t, t)}{K_{n}(z, z)}\right)^{1 / 2}\left[1-\frac{K_{n}^{*}(z, z)}{K_{n}(z, z)}\right]^{1 / 2} .
\end{aligned}
$$

Proof

The idea is to estimate the $L_{2}$ norm of $K_{n}-K_{n}^{*}$ over $G$, and then to use 
Christoffel function estimates. Now

$$
\begin{aligned}
& \int_{G}\left|\left(K_{n}-K_{n}^{*}\right)(z, t)\right|^{2} d \mu(t) \\
= & \int_{G}\left|K_{n}(z, t)\right|^{2} d \mu(t)-2 \operatorname{Re} \int_{G}\left(\overline{K_{n}} K_{n}^{*}\right)(z, t) d \mu(t) \\
& +\int_{G}\left|K_{n}^{*}(z, t)\right|^{2} d \mu(t) \\
= & K_{n}(z, z)-2 K_{n}^{*}(z, z)+\int_{G}\left|K_{n}^{*}(z, t)\right|^{2} d \mu(t),
\end{aligned}
$$

by the reproducing kernel property. As $\mu \leq \mu^{*}$, we also have

$$
\int_{G}\left|K_{n}^{*}(z, t)\right|^{2} d \mu(t) \leq \int_{G}\left|K_{n}^{*}(z, t)\right|^{2} d \mu^{*}(t)=K_{n}^{*}(z, z) .
$$

So

$$
\begin{aligned}
& \int_{G}\left|\left(K_{n}-K_{n}^{*}\right)(z, t)\right|^{2} d \mu(t) \\
\leq & \left(K_{n}-K_{n}^{*}\right)(z, z) .
\end{aligned}
$$

Next for any polynomial $P$ of degree $\leq n-1$, we have the Christoffel function estimate

$$
|P(t)| \leq K_{n}^{1 / 2}(t, t)\left(\int_{G}|P|^{2} d \mu\right)^{1 / 2} .
$$

Applying this to the polynomial $P(t)=\left(K_{n}-K_{n}^{*}\right)(z, t)$, and using (4.2) gives the result.

From this we readily deduce:

\section{Lemma 4.2}

Assume that $\mu, \mu^{*}$ are regular measures on $G$, and that $G, J, \mu$ are as in Theorem 1.1 or 1.3. Assume $\mu^{*}$ is also absolutely continuous with respect to planar measure in a relatively open subset of $\bar{G}$ set containing $J$. Assume that

$$
d \mu=d \mu^{*} \text { in } J
$$

Let $A>0$. Then as $n \rightarrow \infty$,

$$
\left|\left(K_{n}-K_{n}^{*}\right)\left(z_{n}, t_{n}\right)\right| / n^{2}=o(1),
$$

uniformly for all sequences $\left\{z_{n}\right\},\left\{t_{n}\right\} \subset \partial G$ with the following properties: for some $z_{0} \in J$ we have for all $n \geq 1,\left|z_{n}-z_{0}\right| \leq A / n,\left|t_{n}-z_{0}\right| \leq A / n$.

Proof

We initially assume that

$$
d \mu \leq d \mu^{*} \text { in } G \text {. }
$$

Then the inequality (4.1) of Lemma 4.1 holds. Now we set $z=z_{n}$ and $t=t_{n}$ where $\left\{z_{n}\right\},\left\{t_{n}\right\}$ are as above. By Theorem 3.1, uniformly for such 
sequences, $\frac{K_{n}^{*}\left(z_{n}, z_{n}\right)}{K_{n}\left(z_{n}, z_{n}\right)}=1+o(1)$, for $\left(\mu^{*}\right)^{\prime}=\mu^{\prime}=w$ in $J$. Moreover,

$$
K_{n}\left(z_{n}, z_{n}\right) \sim K_{n}\left(t_{n}, t_{n}\right) \sim n^{2}
$$

so uniformly for such $\left\{z_{n}\right\},\left\{t_{n}\right\},(4.1)$ gives

$$
\left|\left(K_{n}-K_{n}^{*}\right)\left(z_{n}, t_{n}\right)\right| / n^{2}=o(1) .
$$

Now we drop the extra hypothesis (4.4). Define a measure $\nu$ by $\nu=\mu=\mu^{*}$ in $J$; and in $G \backslash J$, let

$$
d \nu(t)=\max \left\{\operatorname{dist}(t, J), w(t), w^{*}(t)\right\} d A(t)+d \mu_{s}(t)+d \mu_{s}^{*}(t),
$$

where $w, w^{*}$ and $\mu_{s}, \mu_{s}^{*}$ are respectively the absolutely continuous and singular components of $\mu, \mu^{*}$. Then $\mu \leq \nu$ and $\mu^{*} \leq \nu$, and $\nu$ is regular as its absolutely continuous component is positive in $G$. Moreover, $\nu$ is absolutely continuous in an open subset of $G$ whose boundary contains $J$, and $w^{*}=w$ in $J$. The case above shows that the reproducing kernels for $\mu$ and $\mu^{*}$ have the same asymptotics as that for $\nu$, in the sense of (4.3), and hence the same asymptotics as each other.

We next approximate $\mu$ of Theorem 1.1 by a multiple $\mu^{\#}$ of Lebesgue measure on $G$, and then prove Theorem 1.1. Recall that $\tilde{K}_{n}$ is the normalized kernel, given by (1.4).

\section{Lemma 4.3}

Let $G, J, \mu$ be as in Theorem 1.1 or 1.3. Let $A>0, \varepsilon \in\left(0, \frac{1}{2}\right)$ and choose $\delta>0$ such that (3.3) holds. Then there exists $n_{0}$ such that for $n \geq n_{0}$, $z_{0} \in J$ and $z, t \in B\left(z_{0}, \frac{\delta}{2}\right) \cap \partial G$,

$$
\left|\left(\tilde{K}_{n}-\tilde{K}_{n}^{C}\right)(z, t)\right| / n^{2} \leq C \sqrt{\varepsilon},
$$

Here $C$ is independent of $n, z, t, z_{0}, \varepsilon, \delta$.

\section{Proof}

Fix $z_{0} \in J$ and let $w^{\#}$ be the scaled Legendre weight

$$
w^{\#}=w\left(z_{0}\right) \text { in } G \text {. }
$$

Note that

$$
K_{n}^{\#}(\cdot, \cdot)=\frac{1}{w\left(z_{0}\right)} K_{n}^{C}(\cdot, \cdot) .
$$

Because of our localization result Lemma 4.2, we may replace $d \mu$ by $w^{*}(t) d A(t)$, where

$$
w^{*}=w \text { in } B\left(z_{0}, \delta\right)
$$

and

$$
w^{*}=w\left(z_{0}\right) \text { in } G \backslash B\left(z_{0}, \delta\right),
$$

without affecting the asymptotics for $K_{n}(z, t) / n^{2}$ in the ball $B\left(z_{0}, \frac{\delta}{2}\right)$. (Note that $\varepsilon$ and $\delta$ play no role in Lemma 4.2). So in the sequel, we assume 
that $w=w\left(z_{0}\right)=w^{\#}$ in $G \backslash B\left(z_{0}, \delta\right)$, while not changing $w$ in $B\left(z_{0}, \delta\right)$. Observe that (3.3) implies that

$$
(1+\varepsilon)^{-1} \leq \frac{w}{w^{\#}} \leq 1+\varepsilon, \text { in } G .
$$

Let

$$
w_{1}=(1+\varepsilon) w .
$$

Then $w_{1} \geq w^{\#}$ in $G$, and if $K_{n}^{1}$ denotes the kernel function for $w_{1}$, we have

$$
K_{n}^{1}(\cdot, \cdot)=\frac{1}{1+\varepsilon} K_{n}(\cdot, \cdot)
$$

By Lemma 4.1 , for all $u, v \in \mathbb{C}$,

$$
\begin{aligned}
& \left|K_{n}^{\#}(u, v)-K_{n}^{1}(u, v)\right| / K_{n}^{\#}(u, u) \\
\leq & \left(\frac{K_{n}^{\#}(v, v)}{K_{n}^{\#}(u, u)}\right)^{1 / 2}\left[1-\frac{K_{n}^{1}(u, u)}{K_{n}^{\#}(u, u)}\right]^{1 / 2} .
\end{aligned}
$$

Here

$$
\frac{K_{n}^{1}(u, u)}{K_{n}^{\#}(u, u)}=\frac{1}{1+\varepsilon} \frac{K_{n}(u, u)}{K_{n}^{\#}(u, u)} \geq \frac{1}{(1+\varepsilon)^{2}}
$$

in view of (4.7). Then (4.6) and (4.8) give for all $z, t \in B\left(z_{0}, \frac{\delta}{2}\right) \cap \partial G$,

$$
\begin{aligned}
& \left|K_{n}^{\#}(z, t)-\frac{1}{1+\varepsilon} K_{n}(z, t)\right| / K_{n}^{\#}(z, z) \\
\leq & \left(\frac{K_{n}^{C}(t, t)}{K_{n}^{C}(z, z)}\right)^{1 / 2}\left[1-\frac{1}{(1+\varepsilon)^{2}}\right]^{1 / 2} .
\end{aligned}
$$

Using that $K_{n}^{\#}(z, t)=O\left(n^{2}\right)$ and $K_{n}^{\#}(z, z) \geq C n^{2}$, we obtain for all $z, t \in$ $B\left(z_{0}, \frac{\delta}{2}\right) \cap \partial G$,

$$
\left|\left(K_{n}-K_{n}^{\#}\right)(z, t)\right| / n^{2} \leq C \sqrt{\varepsilon},
$$

so (4.6) gives

$$
\left|w\left(z_{0}\right) K_{n}(z, t)-K_{n}^{C}(z, t)\right| / n^{2} \leq C \sqrt{\varepsilon} .
$$

Next, by (3.3), we have for $z, t \in B\left(z_{0}, \frac{\delta}{2}\right) \cap G$,

$$
(1+\varepsilon)^{-1} \leq \frac{w(z)^{1 / 2} w(t)^{1 / 2}}{w\left(z_{0}\right)} \leq 1+\varepsilon .
$$


Then for such $z, t$ and $z_{0}$,

$$
\begin{aligned}
& \left|\left(\tilde{K}_{n}-\tilde{K}_{n}^{C}\right)(z, t)\right| / n^{2} \\
= & \left|\frac{w(z)^{1 / 2} w(t)^{1 / 2}}{w\left(z_{0}\right)} w\left(z_{0}\right) K_{n}(z, t)-K_{n}^{C}(z, t)\right| / n^{2} \\
\leq & \left|\frac{w(z)^{1 / 2} w(t)^{1 / 2}}{w\left(z_{0}\right)}-1\right| w\left(z_{0}\right)\left|K_{n}(z, t)\right| / n^{2}+\left|w\left(z_{0}\right) K_{n}(z, t)-K_{n}^{C}(z, t)\right| / n^{2} \\
\leq & C \sqrt{\varepsilon},
\end{aligned}
$$

by (4.9).

Our last lemma is a growth estimate for polynomials:

\section{Lemma 4.4}

Let $\eta>0, M>0$. There exists $C>0$ such that for $n \geq 1$ and polynomials $P$ of degree $\leq n$,

$$
|P(z)| \leq C \sup _{J(\eta) \cap \partial G}|P|
$$

for all $z$ with $\operatorname{dist}(z, J \cap \partial G) \leq \frac{M}{n}$.

\section{Proof}

We use the fact that $J(\eta) \cap \partial G$ consists of finitely many smooth arcs of length at least $2 \eta$, so that the Green's function for the complement of the set is well behaved. More precisely, let $g(z)$ denote the Green's function for $\mathbb{C} \backslash(J(\eta) \cap \partial G)$ with pole at $\infty$. Since $\Gamma=\partial G \in C(1, \alpha)$ with $\alpha>\frac{1}{2}$, each arc of $J(\eta) \cap \partial G$ is smooth and of length $\geq 2 \eta$. Then for $z \in \mathbb{C} \backslash(J(\eta) \cap \partial G)$ with $\operatorname{dist}(z, J) \leq \eta / 2$,

$$
g(z) \leq \operatorname{Cdist}(z, J(\eta) \cap \partial G) .
$$

To see this, we use the classic representation of the Greens function in terms of the equilibrium potential for $J(\eta) \cap \partial G$, and the fact that the equilibrium density is continuous, and so bounded, in $J(\eta / 2) \cap \partial G$. See, for example, $[24$, p. 216]. We now use the Bernstein-Walsh inequality

$$
|P(z)| \leq e^{n g(z)} \sup _{J(\eta) \cap \partial G}|P|
$$

and the estimate above for the Green's function, which gives

$$
g(z) \leq C \operatorname{dist}(z, J \cap \partial G) \leq C \frac{M}{n} .
$$

\section{Proof of Theorems $\mathbf{1 . 1}$ and $\mathbf{1 . 3}$}

Let $A, \varepsilon>0$. Choose $\delta>0$ such that (3.3) holds. Now cover $J$ by, say, $L$ balls $B\left(\hat{z}_{j}, \frac{\delta}{2}\right), 1 \leq j \leq L$, each of diameter $\delta$. For each $j$, there 
exists a threshhold $n_{0}=n_{0}(j)$ for which (4.5) holds for $n \geq n_{0}(j)$ and $z, t \in B\left(\hat{z}_{j}, \frac{\delta}{2}\right) \cap \partial G$. Let $n_{1}$ denote the largest of these. Then we obtain, for $n \geq n_{1}, z_{0} \in J, z, t \in B\left(z_{0}, \frac{\delta}{2}\right) \cap \partial G$,

$$
\left|\left(\tilde{K}_{n}-K_{n}^{C}\right)(z, t)\right| / n^{2} \leq C \sqrt{\varepsilon} .
$$

It follows that if $\left\{z_{n}\right\},\left\{t_{n}\right\}$ are sequences in $\partial G$ such that for some $M>0$ and $z_{0} \in J$, we have $\left|z_{n}-z_{0}\right| \leq \frac{M}{n}$ and $\left|t_{n}-z_{0}\right| \leq \frac{M}{n}$ for all $n$, then

$$
\lim _{n \rightarrow \infty}\left|\left(\tilde{K}_{n}-K_{n}^{C}\right)\left(z_{n}, t_{n}\right)\right| / n^{2}=0 .
$$

In particular, suppose that for some given $a, b$,

$$
z_{n}=z_{0}+\frac{a_{n}}{n} \text { and } t_{n}=z_{0}+\frac{b_{n}}{n}, \text { with } \lim _{n \rightarrow \infty} a_{n}=a \text { and } \lim _{n \rightarrow \infty} b_{n}=b .
$$

From (4.11) and the uniform convergence in Theorem 2.1(c) for Carleman polynomials, we have

$$
\begin{aligned}
\lim _{n \rightarrow \infty} \frac{K_{n}\left(z_{0}+\frac{a_{n}}{n}, z_{0}+\frac{b_{n}}{n}\right)}{K_{n}\left(z_{0}, z_{0}\right)} & =\lim _{n \rightarrow \infty} \frac{\tilde{K}_{n}\left(z_{0}+\frac{a_{n}}{n}, z_{0}+\frac{b_{n}}{n}\right)}{\tilde{K}_{n}\left(z_{0}, z_{0}\right)} \\
& =\lim _{n \rightarrow \infty} \frac{K_{n}^{C}\left(z_{0}+\frac{a_{n}}{n}, z_{0}+\frac{b_{n}}{n}\right)}{K_{n}^{C}\left(z_{0}, z_{0}\right)} \\
& =H\left(a \phi^{\prime}\left(z_{0}\right) \overline{\phi\left(z_{0}\right)}+\overline{b \phi^{\prime}\left(z_{0}\right)} \phi\left(z_{0}\right)\right) .
\end{aligned}
$$

We claim that then this implies for the given $a, b$,

$$
\lim _{n \rightarrow \infty} \frac{K_{n}\left(z_{0}+\frac{a}{n}, z_{0}+\frac{b}{n}\right)}{K_{n}\left(z_{0}, z_{0}\right)}=H\left(a \phi^{\prime}\left(z_{0}\right) \overline{\phi\left(z_{0}\right)}+\overline{b \phi^{\prime}\left(z_{0}\right)} \phi\left(z_{0}\right)\right) .
$$

This will follow if we can show that $\left\{\frac{K_{n}\left(z_{0}+\frac{s}{n}, z_{0}+\frac{t}{n}\right)}{K_{n}\left(z_{0}, z_{0}\right)}\right\}_{n}$ is a normal family for $s, t$ in compact subsets of the plane. To show the latter, recall from Theorem 3.1(b) and Cauchy-Schwarz,

$$
\sup _{u, v \in J(\eta) \cap \partial G}\left|K_{n}(u, v)\right| \leq C n^{2} .
$$

Lemma 4.4 gives that for all $u, v$ with $\operatorname{dist}(u, J \cap \partial G) \leq \frac{M}{n}$ and $\operatorname{dist}(v, J \cap \partial G) \leq$ $\frac{M}{n}$,

$$
\left|K_{n}(u, v)\right| \leq C n^{2}\left(\leq C K_{n}\left(z_{0}, z_{0}\right)\right) .
$$

The desired normality then follows. Finally, we can choose sequences of infinitely many distinct $a, b$ with a finite limit point for which there exist sequences $\left\{z_{n}\right\},\left\{t_{n}\right\}$ in $J(\eta) \cap \partial G$ with the properties (4.11) and (4.12). (In fact, there is a continuum of such $a, b)$. The normality of $\left\{\frac{K_{n}\left(z_{0}+\frac{a}{n}, z_{0}+\frac{b}{n}\right)}{K_{n}\left(z_{0}, z_{0}\right)}\right\}_{n}$ and convergence continuation theorems then show that (4.13) holds uniformly for $a, b$ in compact subsets of the plane. 


\section{Proof of Corollary 1.4}

Exactly as in the proof of Corollary 2.2, we have the Taylor series identity (2.8) for $K_{n}$ instead of $K_{n}^{C}$. Moreover, as at (4.13), we have the uniform convergence of $\frac{1}{K_{n}\left(z_{0}, z_{0}\right)} K_{n}\left(z_{0}+\frac{a}{n}, z_{0}+\frac{b}{n}\right)$ for $a, b$ in compact subsets of the complex plane. Recalling the identity (2.9) from Theorem 2.1, the result now follows, for each fixed $z \in J$. However, the uniformity in $z$ still must be proved separately. For this, we just use the uniform boundedness in $z$ also.

\section{Acknowledgement}

The author is grateful for useful discussions about peak polynomial points with Igor Pritsker, as well as references that he provided.

\section{REFERENCES}

[1] V. Andrievskii and I. Pritsker, personal communication.

[2] A. Avila, J. Last, and B. Simon, Bulk universality and clock spacing of zeros for ergodic Jacobi matrices with a.c. spectrum, submitted.

[3] P. Deift, Orthogonal Polynomials and Random Matrices: A Riemann-Hilbert Approach, Courant Institute Lecture Notes, Vol. 3, New York University Pres, New York, 1999.

[4] P. Deift, T. Kriecherbauer, K. T-R. McLaughlin, S. Venakides and X. Zhou, Uniform Asymptotics for Polynomials Orthogonal with respect to Varying Exponential Weights and Applications to Universality Questions in Random Matrix Theory, Communications in Pure and Applied Maths., 52(1999), 1335-1425.

[5] M. Findley, Universality for Regular Measures satisfying Szeg"o's Condition, J. Approx. Theory, 155(2008), 136-154.

[6] D. Gaier, Lectures on Complex Approximation, Boston, Birkhauser, 1987 (transl. by R. McLaughlin).

[7] B. Gustafsson, M. Putinar, E.B. Saff, and N. Stylianopoulos, Les polynomes orthogonaux de Bergman sur un archipel, Comptes Rendus Acad. Sci. Paris Ser. I, 346 (2008), no. 9-10, pp. 499-502.

[8] B. Gustafsson, M. Putinar, E.B. Saff, and N. Stylianopoulos, Bergman Polynomials on an archipelago: estimates, zeros and shape construction, to appear in Advances in Mathematics.

[9] A.B. Kuijlaars and M. Vanlessen, Universality for Eigenvalue Correlations from the Modified Jacobi Unitary Ensemble, International Mathematics Research Notices, 30(2002), 1575-1600.

[10] Eli Levin and D.S. Lubinsky, Universality Limits Involving Orthogonal Polynomials on the Unit Circle, Computational Methods and Function Theory, 7(2007), 543-561.

[11] Eli Levin and D.S. Lubinsky, Universality Limits in the Bulk for Varying Measures, Advances in Mathematics, 219(2008), 743-779.

[12] Eli Levin, E.B. Saff and N.S. Stylianopoulos, Zero Distribution of Bergman Orthogonal Polynomials for Certain Planar Domains, Constr. Approx., 19(2003), 411-435.

[13] D.S. Lubinsky, A New Approach to Universality Limits involving Orthogonal Polynomials, Annals of Mathematics, 170(2009), 915-939.

[14] D.S. Lubinsky, A New Approach to Universality Limits at the Edge of the Spectrum, Contemporary Mathematics (60th Birthday of Percy Deift), 458(2008), 281-290.

[15] D.S. Lubinsky, Universality Limits in the Bulk for Arbitrary Measures with Compact Support, J. d' Analyse de Mathematique, 106(2008), 373-394. 
[16] A. Mate, P. Nevai, V. Totik, Szego"'s Extremum Problem on the Unit Circle, Annals of Mathematics, 134(1991), 433-453.

[17] K. T.-R. McLaughlin and P. Miller, The $\bar{\partial}$ steepest descent method for orthogonal polynomials on the real line with varying weights, Intern. Math. Res. Notices, 2008, Article ID rnn075, pages 1-66.

[18] E. Mina-Diaz, An Asymptotic Integral Representation for Carleman Orthogonal Polynomials, International Mathematics Research Notices (2008) Vol. 2008 : article ID rnn065, 38 pages.

[19] E. Mina-Diaz, E.B. Saff, and N.S. Stylianopoulos, Zero Distributions for Polynomials Orthogonal with Weights over Certain Planar Domains, Comp. Methods and Function Theory, 5(2005), 185-221.

[20] B. Nagy and V. Totik, Sharpening of Hilbert's Lemniscate Theorem, J. d' Analyse de Mathematique, 96(2005), 191-223.

[21] P. Nevai, Orthogonal Polynomials, Memoirs of the AMS no. 213 (1979).

[22] P. Nevai, Geza Freud, Orthogonal Polynomials and Christoffel Functions: A Case Study, J. Approx. Theory, 48(1986), 3-167.

[23] E. B. Saff, Orthogonal Polynomials from a Complex Perspective, (in) Orthogonal Polynomials: Theory and Practice (ed. P. Nevai), Kluwer, Dordrecht, 1990, pp. 363393.

[24] E.B. Saff and V. Totik, Logarithmic Potentials with External Fields, Springer, New York, 1997.

[25] B. Simon, Two Extensions of Lubinsky's Universality Theorem, J. d' Analyse de Mathematique, 105 (2008), 345-362.

[26] B. Simon, The Christoffel-Darboux Kernel, (in) Perspectives in PDE, Harmonic Analysis and Applications, a volume in honor of V.G. Maz'ya's 70th birthday, Proceedings of Symposia in Pure Mathematics, 79 (2008), 295-335

[27] H. Stahl and V. Totik, General Orthogonal Polynomials, Cambridge University Press, Cambridge, 1992.

[28] P.K. Suetin, Polynomials Orthogonal over a Region and Bieberbach Polynomials, Proc. Steklov. Inst.Math., no. 100, American Math. Soc. Translations, Providence, 1974.

[29] V. Totik, Asymptotics for Christoffel Functions for General Measures on the Real Line, J. d' Analyse de Mathematique, 81(2000), 283-303.

[30] V. Totik, Universality and fine zero spacing on general sets, Arkiv för Matematik, 47(2009), 361-391.

[31] V. Totik, Christoffel Functions on Curves and Domains, to appear in Trans. Amer. Math. Soc.

School of Mathematics, Georgia Institute of Technology, Atlanta, GA 30332-0160, USA., LUBINSKY@MATH.GATECH.EDU 